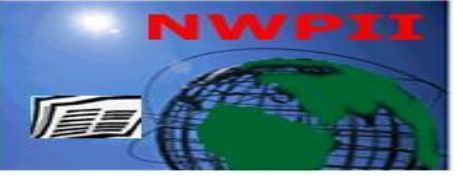

American Journal of Biomedical Sciences

ISSN: 1937-9080

nwpii.com/ajbms

\title{
Interference of Human Spermatozoal Motility by Live Staphylococcus aureus
}

\author{
Siftjit Kaur $^{1}$, Vijay Prabha* ${ }^{*}$, Geeta Shukla ${ }^{1}$ and Abha Sarwal ${ }^{2}$ \\ ${ }^{1}$ Department of Microbiology, Panjab University, Chandigarh, India. \\ ${ }^{2}$ Department of Pathology, Government Multi Speciality Hospital, Chandigarh, India. \\ *Corresponding author \\ Dr. (Mrs) Vijay Prabha \\ Associate Professor \\ Department of Microbiology \\ Panjab University \\ Chandigarh-160014. India \\ Telephone: 91-172-2534144 \\ Fax: 91-172-2541770 \\ E mail: satishvijay11@yahoo.com
}

Received: 10 September 2009; | Revised: 30 November 2009; | Accepted: 10 December 2009

\begin{abstract}
The aim of this work was to investigate the effect of live Staphylococcus aureus commonly present in the cervix of females on sperm motility and agglutination in vitro. Highly motile samples of spermatozoa (40 x $10^{6} / \mathrm{ml}$ ) from normozoospermic males were co-incubated for $4 \mathrm{~h}$ with $10^{7}$ cells $/ \mathrm{ml}$ of live S. aureus. After 30 minutes, 1, 2 and 4h of incubation, sperm motility and agglutination was checked by light microscopic examination (X 400). From the onset, it was apparent that the live bacteria reduced the motility of spermatozoa. The promptness with which the decreased motility was observed to occur in the live $S$. aureus group was striking. After 30 minutes of mixing the ejaculate with the live bacteria, decrease in motility and clumping of the spermatozoa was noted. Interestingly, no agglutination and immobilization was observed in other experiments in which the bacteria were killed by heat, lysozyme or antibiotics and mixed with the ejaculate. Certainly, it seems evident that live $S$. aureus can significantly depress the motility and agglutinate human spermatozoa in vitro. The mechanism by which this alteration takes place is not obvious, however, detrimental effect on sperm motility by live staphylococci may be an as yet unrecognized component of fertility problems.
\end{abstract}

Keywords: Staphylococcus aureus; sperm immobilization; heat; lysozyme; antibiotics; scanning electron microscopy.

\section{Introduction}

Infections of the male genitourinary tract represent a significant health care problem and account for almost $15 \%$ of cases of male infertility

Am. J. Biomed. Sci. 2010, 2(1), 91-97; doi: 10.5099/aj100100091
(Gdoura et al., 2007). Infections can affect different sites of the male reproductive tract, such as the testis, the epididymus and male accessory sex glands (Diemer et al., 2000; Golshani et al., 2006). Spermatozoa subsequently can be affected

(C) 2010 by NWPII. All rights reserved. 
by infections at different points in their development and maturation. Acute and chronic infections can compromise spermatogenesis, resulting in quantitative and qualitative reductions. Direct interactions with pathogenic bacteria represents another possibility for infectious impact on spermatozoa (Khalili and Yazdi, 2001; Golshani et al., 2006).

Among bacterial species that interact with spermatozoa are well known causative pathogens of genitourinary infections such as Escherichia coli, Ureaplasma urealyticum, Mycoplasma hominis and Chlamydia trachomatis (Kohn et al., 1998, Hosseinzadeh et al., 2001, Gdoura et al., 2007). E. coli probably represents the most frequently isolated microorganism in genitourinary infections (Weidner et al., 1991). E. coli rapidly adheres to human spermatozoa in vitro, resulting in agglutination of spermatozoa (Diemer et al., 2003). Agglutination of spermatozoa was produced only by live pathogenic E. coli whereas killed bacteria failed to do so. Liu et al. (2002) while studying other uropathogenic microorganisms found significant decrease in sperm motility when spermatozoa were coincubated with $S$. aureus. Jiang and $\mathrm{Lu}$ (1996) reported $S$. aureus as the predominant flora in infertile men with a significant decrease in sperm motility. Based on these observations and our observation that Staphylococcus aureus, one of the predominant flora in the infertile men and commonly present in the cervix of females immobilizes the spermatozoa (Ohri and Prabha, 2005), we devised a series of simple experiments to determine whether decrease in motility is associated with live bacteria or killed bacteria can also bring about the same change.

\section{Materials and methods}

\subsection{Semen samples}

Spermatozoa were obtained from 10 healthy donors and men undergoing evaluation of fertility at PGIMER, Chandigarh, India, by masturbation following a $24 \mathrm{~h}$ continence period. Only ejaculates showing normal sperm parameters according to World Health Organization criteria (1999) were used.

Am. J. Biomed. Sci. 2010, 2(1), 91-97; doi: 10.5099/aj100100091

\subsection{Microorganism}

The bacterial isolates used in the present study were taken from the cervices of 10 women with unexplained infertility who were seen at the Department of Obstetrics and Gynecology, Government Multi Speciality Hospital, Sector-16, Chandigarh, India. Before taking the samples, the patients' recent medical history was taken into consideration. The cervical samples were taken from only those females who had not had any antibiotic intake for at least a week. The sample was taken only at that time when there was no clinical incidence of cervical infection. Therefore, all the cervical isolates which were obtained were considered to be normal flora. Swab samples were taken from the cervical area by the standard technique (Hok et al., 1967). The samples were directly spread by streaking on sheep blood agar plates and the plates were incubated aerobically at $37^{\circ} \mathrm{C}$ for $24-48 \mathrm{~h}$. In total 10 isolates were obtained. The isolates were identified according to Bergey's Manual of Determinative Bacteriology (1994). Screening of 10 isolates for interaction with human sperm identified only one isolate, $S$. aureus as an inducer of sperm agglutination. Therefore, further experiments were carried out with this isolate.

\subsection{Killing of bacteria}

Killing of bacteria was carried out by using three different methods viz. heat, lysozyme and antibiotic treatment.

Heat treatment: Aliquots of $2 \mathrm{ml}$ of $72 \mathrm{~h}$ old culture of $S$. aureus were exposed to different temperatures viz. $40,50,60,80$ and $100^{\circ} \mathrm{C}$ for 10 and 30 minutes and checked for non- viability by subsequent culturing on nutrient agar plates.

Lysozyme treatment: Aliquots of $2 \mathrm{ml}$ of $72 \mathrm{~h}$ old culture of $S$. aureus were incubated with different concentrations $\left(10-1000 \mu \mathrm{g} \mathrm{ml}^{-1}\right)$ of hen egg white lysozyme (Hi-Media Laboratories, Mumbai, India) for different time intervals (2-24h) at $37^{\circ} \mathrm{C}$ in order to find out the best dose time combination for killing of bacteria (Millar, 1987). After completion of incubation, the culture was checked for non- viability by subsequent culturing on nutrient agar plates.

Antibiotic treatment: Eleven antibiotics were chosen for the study according to their common 
use in research and human medicine. They belonged to following groups $\beta$-lactams or penicillins: amoxicillin (Maxheal pharmaceuticals, Maharashtra, India), ampicillin (Maxheal pharmaceuticals, Maharashtra, India), penicillin G (Hindustan Antibiotics Ltd., India), methicillin; macrolides: erythromycin (Shalina Laboratories Ltd., Mumbai, India); aminoglycosides: gentamicin (Maxheal pharmaceuticals, Maharashtra, India), streptomycin (Synbiotics Ltd., Vadodra, India); quinolones: ciprofloxacin (Shalina Laboratories Ltd., Mumbai, India); glycopeptides: vancomycin (VHB Life Sciences, Kerala, India); lincosamides: clindamycin (Pfizer Manufacturing, Belgium) and chloramphenicol (Maxheal pharmaceuticals, Maharashtra, India). Stock solutions $\left(1 \mathrm{mg} \mathrm{ml}^{-1}\right)$ of each antibiotic were prepared using water for injection. $\mathrm{MBC}$ (Minimum Bactericidal Concentration) for each antibiotic was obtained using the standard protocol (Scott, 1989). MBC was defined as a $99.99 \%$ reduction of cell viability with respect to that of initial inoculum.

\subsection{Effect of live and dead bacteria on sperm agglutination}

$0.1 \mathrm{ml}$ volumes of fresh ejaculate were mixed with $0.1 \mathrm{ml}$ of each of the following: 7.2).

(1) Phosphate Buffered Saline (50 mM, pH

(2) $72 \mathrm{~h}$ old live culture of $S$. aureus.

(3) $72 \mathrm{~h}$ old culture of $S$. aureus exposed to $100^{\circ} \mathrm{C}$ for 10 minutes.

(4) $72 \mathrm{~h}$ old culture of $S$. aureus preincubated with lysozyme at a concentration of $1 \mathrm{mg} \mathrm{ml}{ }^{-1}$ for $4 \mathrm{~h}$ at $37^{\circ} \mathrm{C}$ and after incubation removal of lysozyme from the culture by centrifugation and washing the cell pellet twice with PBS (50 mM, pH 7.2) under cold conditions and pellet resuspended in same volume of PBS (Reddy et al., 2004).

(5) $72 \mathrm{~h}$ old culture of $S$. aureus preincubated with the MBC of each of antibiotic for $24 \mathrm{~h}$ and after incubation removal of antibiotic from the culture by centrifugation and washing the cell pellet twice with PBS (50 mM, pH 7.2) under cold conditions and pellet resuspended in same volume of PBS (Reddy et al., 2004).
Following mixing, a drop each of the suspension was placed on a glass slide and examined under the microscope to determine immobilization/agglutination. These examinations were repeated at different intervals for $4 \mathrm{~h}$.

\subsection{Scanning Electron Microscopy (SEM)}

Scanning electron microscopy was used to investigate the adherence of live and dead bacteria on spermatozoa. The sample processing was done according to the standard method (Hafez and

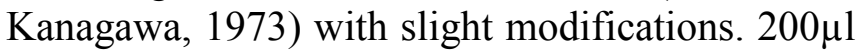
of washed sperm suspension was incubated with $200 \mu 1$ of each live and killed (heat/lysozyme/ antibiotic) $S$. aureus for $4 \mathrm{~h}$. To each tube $4 \mathrm{ml}$ of $2.5 \%$ phosphate buffered gluteraldehyde was added gently with a pasteur pipette. After 30 minutes samples were centrifuged at $500 \mathrm{x}$ g for 10 minutes and washed twice in PBS $(50 \mathrm{mM}, \mathrm{pH}$ 7.2). One drop of fixed and washed spermatozoa was placed on a silver painted adhesive tape mounted on brass stubs and air dried. $100 \AA \hat{~ g o l d ~}$ coating was done on Jeol fine coat ion sputter (JFC-1100) and the specimens were observed.

\section{Results and discussion}

There is disagreement as to the influence of certain microbial infection on male infertility. Several investigators have reported different types of organisms in seminal fluid specimens depending on the methods of examination. Detection of bacteria in semen does not necessarily suggest infection. Since bacterial isolates in seminal fluid may represent contamination or colonization of the uretheral orifice. Staphylococcus aureus has been isolated from $68.2 \%$ of the seminal fluids (Okon et al., 2005) in Maiduguri. Most practitioners discuss the presence of $S$. aureus as mere contamination which is assumed to be of no significance. However, Emokpae et al. (2009) reported that presence of $S$. aureus should not be ignored as it can lead to decrease in the number of spermatozoa, the suppression of their motility, changes in their morphology and fertilizing capacity. Earlier work done by Jiang and Lu, (1996) and Liu et al. (2002) has also shown $S$. 
aureus to be the dominant flora in infertile men with a significant decrease in sperm motility.

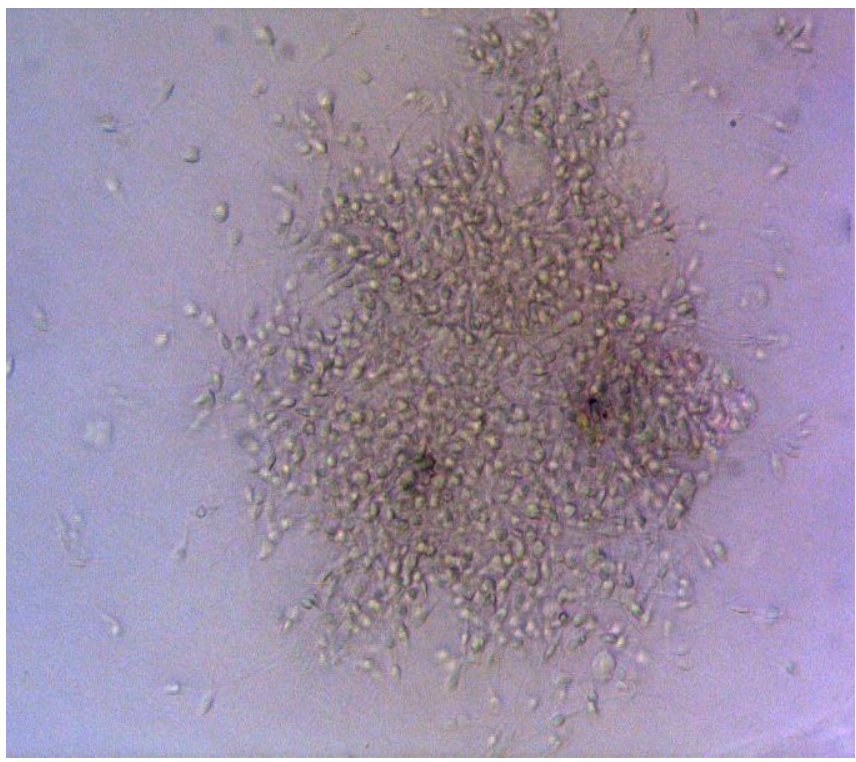

Figure 1a

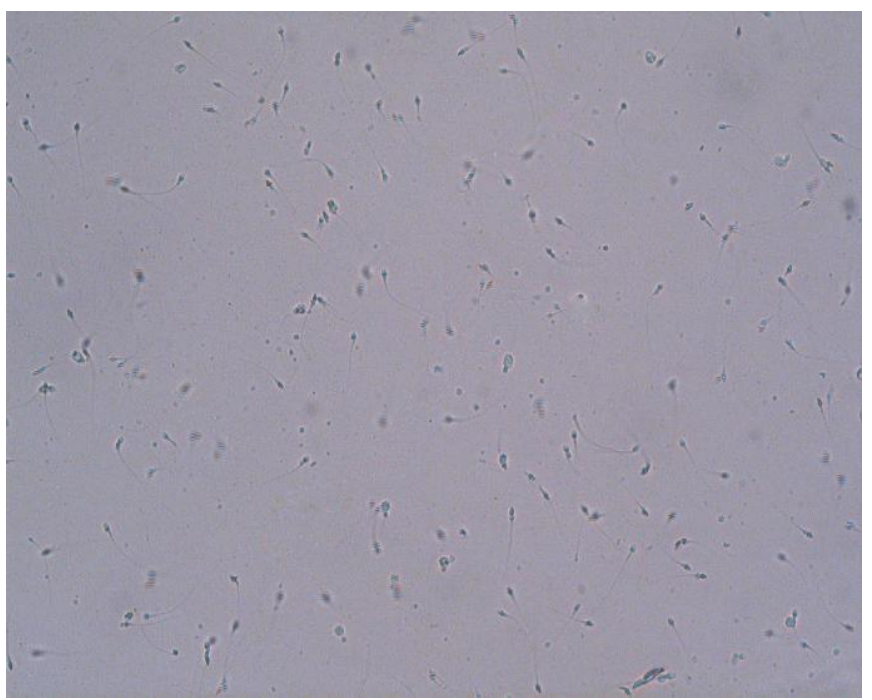

Figure 1b

Figure 1: (a) Agglutination of motile spermatozoa by live $S$. aureus (x 400). (b) No agglutination by dead $S$. aureus (x 200).

In the present studies we have also found that $S$. aureus isolated from the cervix of a woman with unexplained infertility produces profound depression in the motility of human spermatozoa in vitro. Within 30 minutes of mixing of semen with live $S$. aureus, clumping of spermatozoa and dampening of motility was observed. At $4 \mathrm{~h}$ there was complete absence of motility and significant agglutination of spermatozoa (Figure 1a). Interestingly, no agglutination or dampening of motility was noted in those cases (Figure 1b) where the bacteria were killed before mixing with the ejaculate by treatment with heat at $100^{\circ} \mathrm{C}$ for 10 minutes (Figure 2a), lysozyme at a conc. of $1 \mathrm{mg} \mathrm{ml}^{-1}$ for $4 \mathrm{~h}$ (Figure $2 \mathrm{~b}$ ) and various antibiotics (MBC, Table 1).

Table 1: Minimum bactericidal concentration of various antibiotics used to kill bacteria

\begin{tabular}{|c|c|}
\hline Antimicrobial & MBC $(\mu \mathrm{g} / \mathrm{ml})$ \\
\hline Ampicillin & 6 \\
\hline Amoxycillin & 20 \\
\hline Ciprofloxacin & 40 \\
\hline Chloramphenicol & 6 \\
\hline Clindamycin & 8 \\
\hline Erythromycin & 10 \\
\hline Gentamicin & 4 \\
\hline Methicillin & 6 \\
\hline Penicillin G & 10 \\
\hline Streptomycin & 10 \\
\hline Vancomycin & 10 \\
\hline
\end{tabular}

Further to ascertain the mechanism underplaying the agglutination and dampening of spermatozoal motility, scanning electron microscopy was performed. The electron micrographs showed close association between live $S$. aureus and spermatozoa. The bacteria were seen to adhere to tail and head (Figure 3a). Along with bacterial adherence, a prominent morphological defect i.e. the curling of tail was also observed. These results are in accordance to earlier studies carried out by Diemer et al. (2000) wherein they have also reported the adherence of 
E. coli to both heads and tails of spermatozoa. Further, electron microscopic evaluation of spermE. coli interactions revealed multiple and profound alterations involving all superficial structures of spermatozoa, indicating that morphological defects might be accounting for the immobilization of spermatozoa (Diemer et al., 2000).

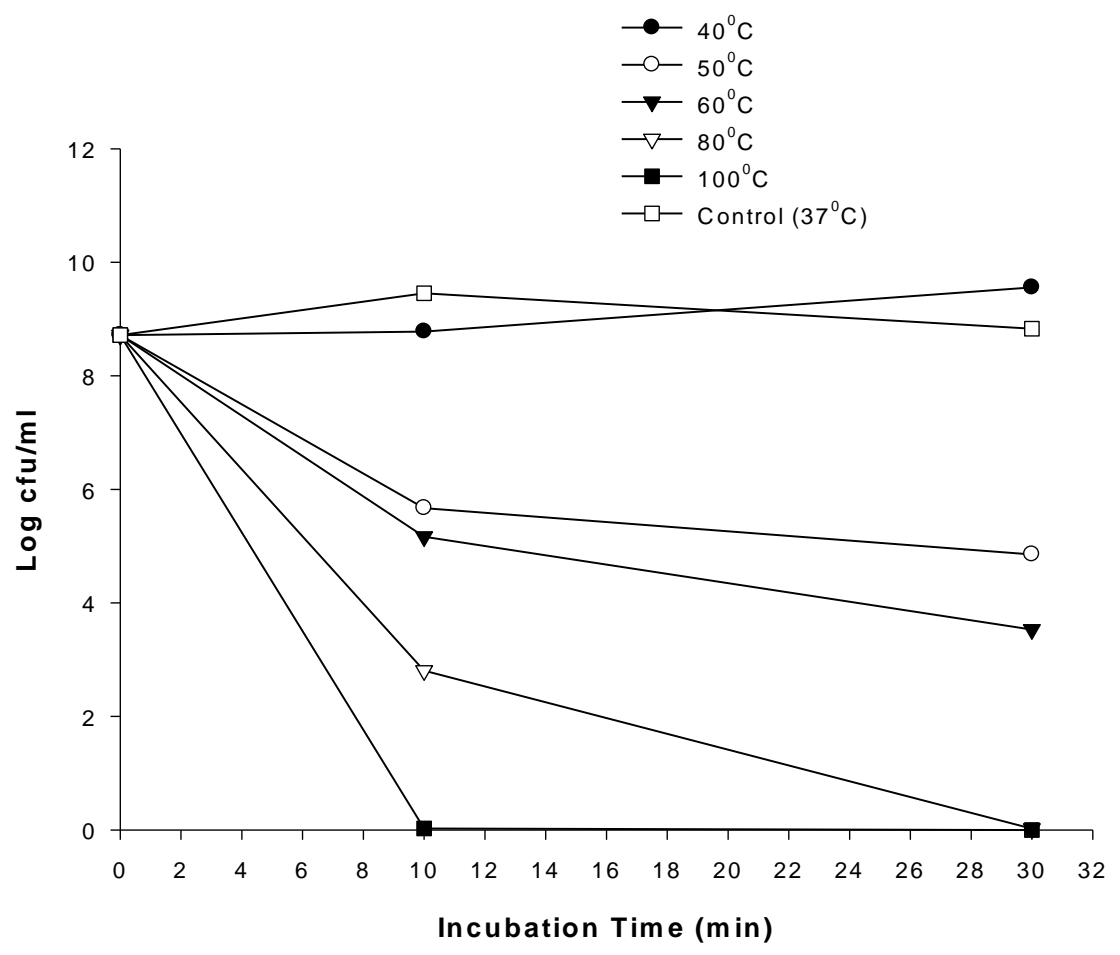

Figure 2a

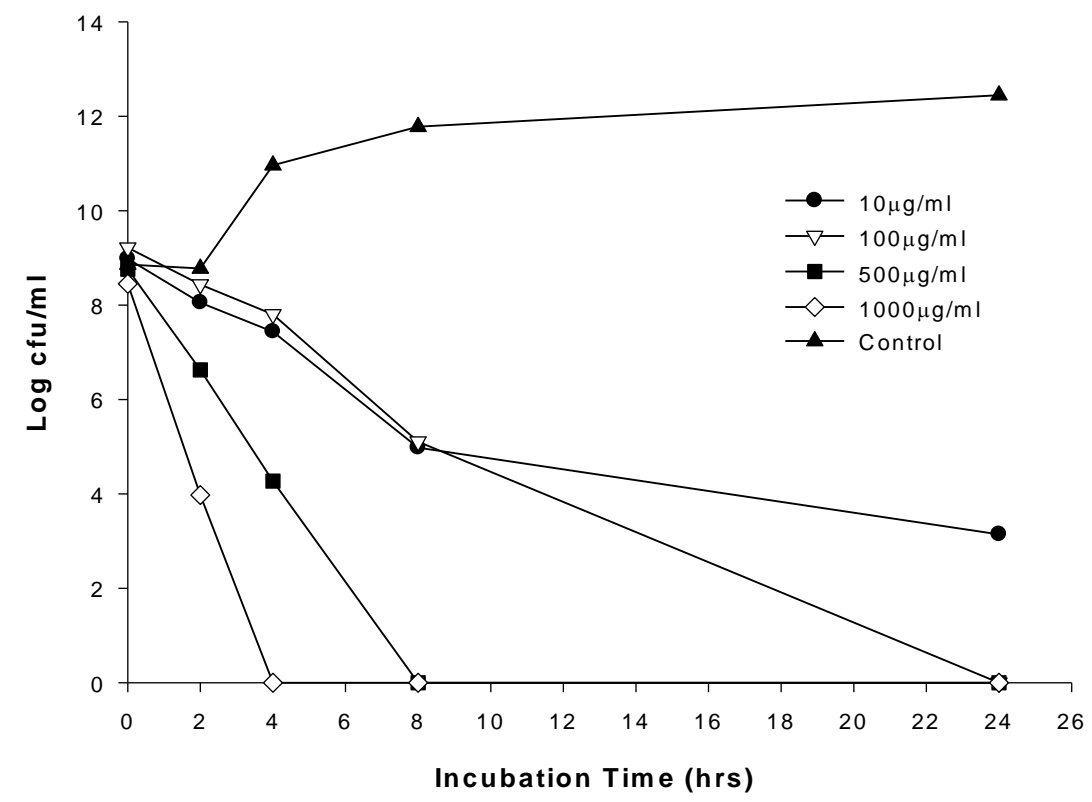

Figure 2b

Figure 2: Viable count plating after treatment of $S$. aureus with (a) heat and (b) lysozyme. 


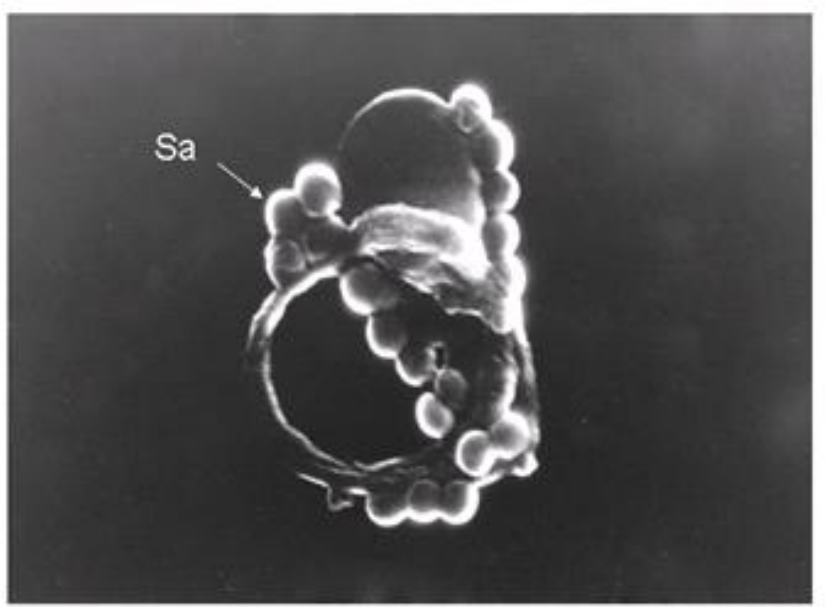

Figure 3a

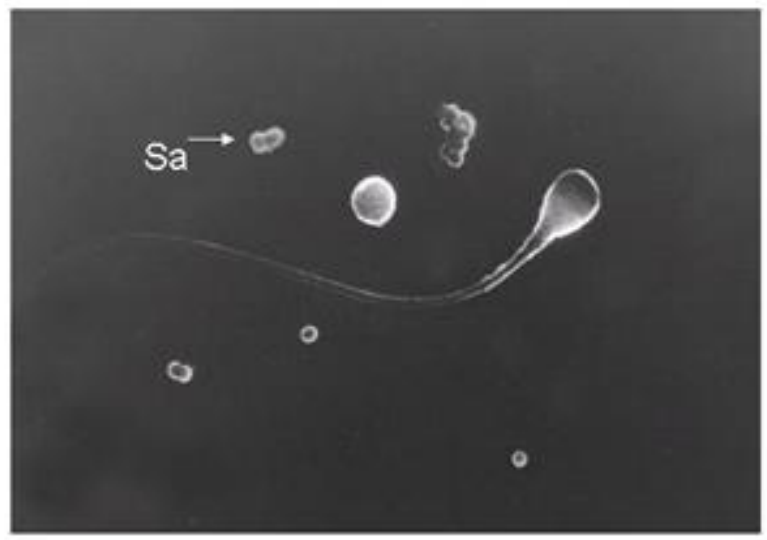

Figure 3b

Figure 3: Scanning electron micrograph showing (a) adherence of live $S$. aureus (Sa) $($ x 8,500) and (b) non-adherence of dead $S$. aureus (Sa), to human sperm (x 2,700)

The dead $S$. aureus neither adhered to spermatozoa nor induced any changes in the morphology (Figure 3b) which might be the reason of causing no immobilization of the spermatozoa. In an earlier study, Hosseinzadeh et al. (2001) have also shown that live Chlamydial elementary bodies (EBs) can have a direct and detrimental effect on sperm physiology whereas heat treatment abolishes the same.

The absence of immobilization and agglutination of spermatozoa upon incubation with killed $S$. aureus could be suggestive of some labile factor present on bacterial cells. However, this mechanism clearly requires further investigation.

Am. J. Biomed. Sci. 2010, 2(1), 91-97; doi: 10.5099/aj100100091

\section{Conclusion}

Live $S$. aureus obtained from cervical cultures agglutinates and immobilizes human spermatozoa in vitro whereas killed bacteria fail to do so.

\section{Acknowledgement}

This work was supported by funds from University Grants Commission (UGC), New Delhi.

\section{References}

1. Bergey's Manual of Determinative Bacteriology (1994) Bergey DH \& Holt GJ. $9^{\text {th }}$ Edition. Lippincott Williams \& Wilkins, Baltimore, Maryland U.S.A.

2. Diemer T, Huwe P, Michelmann HW, Mayer F, Schiefer HG and Wiedner W (2000) Escherichia coli-induced alterations of human spermatozoa. An electron microscopy analysis. Int J Androl 23: 178-186.

3. Diemer T, Huwe P, Ludwig M, SchroederPrintzen I, Michelmann HW, Schiefer HG and Wiedner W (2003) Influence of autogenous leucocytes and Escherichia coli on sperm motility parameters in vitro. Andrologia 35: 100-105.

4. Emokpae MA, Uadia PO and Sadiq NM (2009) Contribution of bacterial infection to male infertility in Nigerians. Online $J$ Health Allied Sci http://www.ojhas.org/issue29/20091-6.htm

5. Gdoura R, Kchaou W, Chaari C, Znazen A, Keskes L, Rebai T and Hammami A (2007) Ureaplasma urealyticum, Ureaplasma parvum, Mycoplasma hominis and Mycoplasma genitalium infections and semen quality of infertile men. BMC Infect Dis 7:129-137.

6. Golshani M, Taheri S, Eslami G, Suleimani Rahbar AA, Fallah F and Gourdazi H (2006) Genital tract infection in asymptomatic infertile men and its effect on semen quality. Ir J Publ Health 35(3):81-84.

7. Hafez ESE and Kanagawa H (1973) Scanning electron microscopy of human, monkey and rabbit spermatozoa. Fertil Steril 24: 776-787.

(C) 2010 by NWPII. All rights reserved. 
8. Hok TT, Leon LK and Tijat NT (1967) Comparative bacteriology of endocervical mucus. Am J Obstet Gynecol 98:781-783.

9. Hosseinzadeh S, Brewis IA, Eley A and Pacey AA (2001) Co-incubation of human spermatozoa with Chlamydia trachomatis serovar E causes premature sperm death. Human Reprod 16: 293-299.

10. Jiang J and Lu DY (1996) Detection of bacteria from semens of infertile males and their seminal parameters. Chin J Androl 10: 196-198.

11. Khalili MB and Sharifi-Yazdi MK (2001) The effect of bacterial infection on the quality of human's spermatozoa. Ir J Publ Health 30: 119-122.

12. Kohn FM, Erdmann I, Oeda T, el Mulla KF, Schiefer HG and Schill WB (1998) Influence of urogenital infections on sperm functions. Andrologia 30:73-80.

13. Liu JH, Li HY, Cao ZG, Duan YF, Li Y and Ye ZQ (2002) Influence of several uropathogenic microorganisms on human sperm motility parameters in vitro. Asian $J$ Androl 4:179-182.

14. Millar M (1987) The susceptibility to lysozyme of $\beta$-lactamase producing and nonproducing derivatives of Staphylococcus aureus strain 1030. J Med Microbiol 23:127132.

15. Ohri M and Prabha V (2005) Isolation of sperm-agglutinating factor from
Staphylococcus aureus isolated from a woman with unexplained infertility. Fertil Steril 84: 1539-1541.

16. Okon KO, Nwaogwu M, Zailani SO and Chana C (2005) Pattern of seminal fluid indices among infertile male partners attending the infertility clinic of University of Maiduguri Teaching Hospital, Maiduguri, Nigeria. Highland Med J 1: 18-23.

17. Reddy KVR, Aranha C, Gupta SM and Yedery RD (2004) Evaluation of antimicrobial peptide nisin as a safe vaginal contraceptive agent in rabbits: in vitro and in vivo studies. Reproduction 128: 117-126.

18. Scott AC (1989) Laboratory control of antimicrobial therapy. In: Mackie and Mac Cartney Practical Medical Microbiology. Eds., Collee, J.G., J.P. Duguid, A.G. Fraser, and B.P. Marmion. Churchill Livingstone Publisher, pp: 161-181.

19. Weidner W, Jantos C, Shiefer HG, Haidl G and Freidrich HJ (1991) Semen parameters in men with and without proven chronic prostatitis. Arch Androl 26:173-183.

20. World Health Organisation, 1999. WHO laboratory manual for the examination of human semen and semen cervical mucus interaction. Cambridge University Inc, Cambridge, UK 